\title{
Mortalidade em pessoas idosas do Distrito Federal, 2008-2018: principais causas e
}

\section{fatores relacionados}

\author{
Mortality in the older adults in the Federal District, 2008-2018: main causes and related factors \\ Mortalidad en ancianos en el Distrito Federal, 2008 - 2018: principales causas y factores \\ relacionados
}

Recebido: 08/12/2021 | Revisado: 15/12/2021 | Aceito: 17/12/2021 | Publicado: 22/12/2021

\author{
Geraldo Marques da Costa \\ ORCID: https://orcid.org/0000-0002-1303-7465 \\ Universidade de Brasília, Brasil \\ E-mail: geraldomarques06@gmail.com \\ Mauro Niskier Sanchez \\ ORCID: https://orcid.org/0000-0002-0472-1804 \\ Universidade de Brasília, Brasil \\ E-mail: mauro.sanchez4@gmail.com \\ Helena Eri Shimizu \\ ORCID: https://orcid.org/0000-0001-5612-5695 \\ Universidade de Brasília, Brasil \\ E-mail: helena.eri.shimizu@gmail.com
}

\begin{abstract}
Resumo
Objetivo: Descrever os óbitos dos idosos do Distrito Federal de 2008 a 2018 e analisar as principais causas e fatores relacionados. Metodologia: Estudo do tipo série temporal sobre mortalidade dos idosos, análise descritiva e comparação de taxas através do teste jonckheere-terpstra. Resultados: A idade média foi 76,51. A maioria dos óbitos ocorreu dentre 80 anos ou mais. Sexo masculino predominou. As principais causas de óbito foram infarto agudo do miocárdio $(6,6 \%)$; pneumonia $(5,2 \%)$; acidente vascular cerebral $(4,1 \%)$; neoplasia maligna dos brônquios ou pulmões $(3 \%)$ e doença pulmonar obstrutiva crônica $(2,6 \%)$. Maior mortalidade entre homens $(\mathrm{p}<0,001)$, por causas cardiovasculares $(p=0,006)$, e em idosos mais velhos $(p<0,001)$ foi estatisticamente significativa. Conclusão: A maioria dos óbitos foi por causas cardiovasculares. Ser homem idoso e maior idade estiveram relacionados a maior mortalidade. Os achados sugerem que o aumento da cobertura de atenção primária pode ter contribuído para o padrão observado.
\end{abstract}

Palavras-chave: Idoso; Mortalidade; Saúde do idoso; Causas de morte; Óbito.

\begin{abstract}
Objective: To describe the deaths of the elderly in the Federal District from 2008 to 2018 and analyze the main causes and related factors. Methodology: Time series study on older adults mortality, descriptive analysis and comparison of rates using the jonckheere-terpstra test. Results: The mean age was 76.51. Most deaths occurred between 80 years of age or older. Male sex predominated. The main causes of death were acute myocardial infarction (6.6\%); pneumonia (5.2\%); stroke (4.1\%); malignant neoplasm of the bronchi or lungs (3\%) and chronic obstructive pulmonary disease $(2.6 \%)$. Higher mortality among men $(\mathrm{p}<0.001)$, from cardiovascular causes $(\mathrm{p}=0.006)$, and in older elderly $(\mathrm{p}<0.001)$ was statistically significant. Conclusion: Most deaths were due to cardiovascular causes. Being an elderly man and being older were related to higher mortality. The findings suggest that the increase in primary care coverage may have contributed to the observed pattern.
\end{abstract}

Keywords Elderly; Mortality; Health of the elderly; Causes of death; Death.

\section{Resumen}

Objetivo: Describir las muertes de ancianos en el Distrito Federal de 2008 a 2018 y analizar las principales causas y factores relacionados. Metodología: Estudio de series de tiempo sobre mortalidad de ancianos, análisis descriptivo y comparación de tasas mediante la prueba de jonckheere-terpstra. Resultados: La edad media fue de 76,51 años. La mayoría ocurrieron entre más de 80 años. Predominó el sexo masculino e infarto agudo do miocardio (6,6\%); neumonía (5,2\%); accidente cerebrovascular $(4,1 \%)$; neoplasia maligna de bronquios o pulmones (3\%) y enfermedad pulmonar obstructiva crónica $(2,6 \%)$. La mayor mortalidad entre los hombres $(\mathrm{p}<0,001)$, por causas cardiovasculares $(\mathrm{p}=0,006)$ y en los ancianos mayores $(\mathrm{p}<0,001)$ fue estadísticamente significativa. Conclusión: Predominaron las muertes por causas cardiovasculares. Ser un hombre mayor y ser mayor se relacionó con una mayor mortalidad. Los hallazgos sugieren que el aumento de la cobertura de atención primaria puede haber contribuido al patrón observado.

Palabras clave: Anciano; Mortalidad; Salud de los ancianos; Causas de muerte; Muerte. 


\section{Introdução}

O envelhecimento da população brasileira é notório. Tal fenômeno contribui para o aumento da prevalência de doenças não transmissíveis. As doenças não transmissíveis representam a principal causa de mortes no Brasil, influenciando a população idosa(Figueiredo et al., 2021).

Essa realidade pode gerar impactos importantes na maior demanda de recursos de saúde. A população mais longeva necessita, com maior frequência, de internações hospitalares, além de requerer períodos mais prolongados de cuidados de saúde. A maior prevalência de doenças crônicas exige consultas médicas frequentes, medicamentos e exames (Veras, 2016). Essa situação reveste-se de riscos, pois, com o passar dos anos, o SUS pode se tornar um sistema insustentável, o que pode comprometer a disponibilidade dos serviços para toda a população(Carvalho \& Paes, 2019), além de poder impactar negativamente na mortalidade da população.

Estudar os óbitos da população de idosos é importante, pois contribui para o conhecimento do estado geral da saúde dessa população. Além disso, a partir dessa análise, é possível conhecer os principais grupos de doenças que contribuem para a mortalidade das pessoas idosas. Nessa mesma linha, evidencia-se a relevância dos estudos de tendências de mortalidade que podem ser importantes diretrizes para auxiliar no enfrentamento e na resolução dos desafios apresentados (Duarte \& Barreto, 2012).

No contexto epidemiológico atual, as tendências de mortalidade apresentam padrões diferentes entre as regiões do Brasil. Mesmo com melhorias no sistema de saúde, ainda são grandes as diferenças entre as regiões com melhores condições quando comparadas àquelas que ainda possuem muitos problemas econômicos e sociais (Paes, 2016).

No Distrito Federal, estudos sobre a mortalidade dos idosos ainda são limitados. Não há municípios no Distrito Federal, mas regiões administrativas. Atualmente são 31 que funcionam praticamente como cidades, sem prefeitos e vereadores, mas com administradores indicados pelo governador.(Drumond et al., 2020) A despeito de ter um dos melhores índices de desenvolvimento humano e a maior renda per capita do país, há importante desigualdade na distribuição de renda. Essas particularidades na organização social e na distribuição de renda pode impactar, de forma particular, nas tendências de mortalidade entre pessoas idosas dessa região

Considerando essas particularidades e as lacunas na produção de estudos de mortalidade nessa região é que se justifica o presente estudo. Assim, o levantamento dessas informações pode contribuir para a elaboração de estratégias de saúde direcionadas para aperfeiçoar o atendimento prestado aos idosos, no sentido de melhorar os indicadores de morbimortalidade. Este estudo teve como objetivo descrever os óbitos dos idosos do Distrito Federal, Brasil, no período de 2008 a 2018 , e conhecer as principais causas e os fatores relacionados: sexo e faixa etária.

\section{Metodologia}

Trata-se de um estudo transversal do tipo série temporal. Esse tipo de estudo permite organizar os dados conforme o tempo observando a ocorrência do desfecho na população e fatores que influenciam nesse contexto.(Antunes \& Cardoso, 2015) Foram estudados os dados de mortalidade das pessoas idosas, maiores de 60 anos, ocorridos no Distrito Federal, de 2008 a 2018. O Distrito Federal está localizado na região Centro-Oeste do Brasil. A população em 2020 está estimada em 3.055 .149 pessoas, e densidade populacional de 444,07 habitantes por $\mathrm{km}^{2}$, conforme estimativas do Instituto Brasileiro de Geografia e Estatística(Distrito Federal | Cidades e Estados $\mid$ IBGE, n.d.).

Foram analisados os dados obtidos na declaração de óbito e disponibilizados na base de dados da Secretaria de Saúde do Distrito Federal. A fim de obter maior precisão dos dados, foram estudados todos os óbitos ocorridos no período (20082018) e excluídos os registros incompletos ou relativos a idosos que não residiam no Distrito Federal. Incluíram-se no estudo todos os idosos residentes e falecidos no Distrito Federal, no período estudado. Para acessar o sistema próprio da Secretaria de 
Saúde do Distrito Federal, houve autorização do órgão. Trata-se de um banco de dados interno, alimentado a partir das declarações de óbito. A extração de dados foi realizada em setembro de 2019 e gerou 86.903 ocorrências, porém restaram 85.835 ocorrências após aplicação dos critérios de exclusão. As variáveis estudadas foram: idade na ocasião do óbito, sexo, estado civil, escolaridade, raça/cor e causa básica do óbito.

A análise de mortalidade foi estimada considerando as principais causas de morte, e os óbitos foram divididos por grupos etários e por sexo. A Classificação Internacional de Doenças (CID) foi o instrumento utilizado na definição das causas de morte registrada na declaração de óbito. Utilizou-se a décima versão, conhecida como CID-10. Os grupos etários foram definidos em 60-69, 70-79 e 80 anos ou mais. Também foi realizada a análise da tendência de mortalidade temporal conforme faixa etária e sexo.

Para a análise de dados, realizou-se a análise descritiva a partir de frequências relativas e absolutas, medidas de tendência central e de dispersão. O cálculo de coeficientes de mortalidade foi estimado por 10.000 habitantes e a taxa de variação foi calculada considerando taxa de mortalidade e a variação no período estudado. Foram construídos gráficos, a fim de analisar o comportamento dos eventos de causas de mortalidade por grupos etários e de sexo durante a série temporal.

A análise de mortalidade por sexo, por faixa etária e por causa do óbito foi realizada utilizando o Teste de tendência de Jonckheere - Terpstra. Esse teste avalia diferenças entre taxas de mortalidade dos grupos definidos pelas variáveis de interesse, além de identificar se as medianas dos grupos estudados apresentam alguma tendência linear.

Este estudo foi baseado exclusivamente em dados secundários obtidos na base da Secretaria de Saúde do Distrito Federal. Foi observado sigilo e anonimato conforme a Resolução do Conselho Nacional de Saúde (CNS) no 466, de 12 de dezembro de 2012. O projeto foi aprovado pelo Comitê de Ética em Pesquisa da Faculdade de Saúde da Universidade de Brasília, parecer no 3.479.132; emitido em 31 de julho de 2019, Certificado de Apresentação para Apreciação Ética (CAAE) n08615719.3.0000.0030 Por ter utilizado apenas dados secundários, o termo de consentimento livre e esclarecido foi dispensado.

\section{Resultados}

Na população estudada, analisando o período compreendido de 2008 a 2018, ocorreram 85.835 óbitos, em pessoas com 60 anos ou mais. Verifica-se aumento progressivo da média da idade do óbito no período estudado (Tabela 1).

Tabela 1 - Distribuição dos óbitos, segundo ano e idade. Distrito Federal, Brasil, 2008 a 2018.

\begin{tabular}{llll}
\hline Ano & $\mathbf{N}^{\circ}$ Óbitos (\%) & Idade média (anos) Desvio Padrão & Coeficiente de Variação (\%) \\
\hline 2008 & $6.539(7,6)$ & $75,63 \pm 9,62$ & 12,72 \\
2009 & $6.623(7,7)$ & $75,79 \pm 9,61$ & 12,66 \\
2010 & $7.231(8,4)$ & $76,26 \pm 9,82$ & 12,87 \\
2011 & $7.486(8,7)$ & $76,22 \pm 9,79$ & 12,84 \\
2012 & $7.624(8,9)$ & $76,38 \pm 9,84$ & 12,89 \\
\hline
\end{tabular}

\begin{tabular}{llll}
\hline Ano & $\mathbf{N}^{\circ}$ Óbitos (\%) & Idade média (anos) Desvio Padrão & Coeficiente de Variação $(\%)$ \\
\hline 2013 & $7.825(9,1)$ & $76,42 \pm 9,87$ & 12,91 \\
2014 & $8.563(10)$ & $76,53 \pm 9,91$ & 12,95 \\
2015 & $8.443(9,8)$ & $76,69 \pm 9,91$ & 12,92 \\
2016 & $8.440(9,8)$ & $76,93 \pm 9,93$ & 12,91 \\
2017 & $9.011(10,5)$ & $77,22 \pm 10,15$ & 13,14 \\
2018 & $8.050(9,4)$ & $77,06 \pm 10,09$ & 13,09 \\
Total & $85.835(100)$ & $76,51 \pm 9,89$ & 12,93 \\
\hline
\end{tabular}


A principal causa de óbito encontrada no presente estudo foi infarto agudo do miocárdio, com 6,6\% das ocorrências. A segunda causa mais comum foi pneumonia $(5,2 \%)$, seguida de acidente vascular cerebral $(4,1 \%)$. Dentre as 10 principais causas, observa-se ainda, em ordem decrescente de ocorrência: câncer de brônquios ou pulmão (3\%), doença pulmonar obstrutiva crônica (2,6\%), sequelas de acidente vascular cerebral (2,2\%), diabetes mellitus $(2,2 \%)$, doença de Alzheimer ( $2,1 \%)$, doença de chagas com comprometimento cardíaco $(2,1 \%)$, e outras doenças cerebrovasculares especificadas $(2,0 \%)$.

A tabela 2 descreve a distribuição dos óbitos ocorridos de acordo com faixa etária, estado civil, e raça/cor, e apresenta os valores absolutos e percentuais.

A maioria dos óbitos ocorreu em idosos do sexo masculino, com 80 anos ou mais, casados e que foram declarados brancos.

Tabela 2 - Distribuição dos óbitos segundo características sociodemográficas dos idosos. Distrito Federal, Brasil, 2008 a 2018.

\begin{tabular}{lll}
\hline & & $\mathbf{N}^{\circ}$ Óbitos (\%) \\
\hline Sexo & Feminino & $42.525(49,5)$ \\
Faixa etária & Masculino & $43.310(50,5)$ \\
& De 60 a 69 anos & $24.267(28,3)$ \\
& De 70 a 79 anos & $28.313(33,0)$ \\
Estado civil & 80 anos e mais & $33.255(38,7)$ \\
& Solteiro & $16.171(18,8)$ \\
& Casado & $33.109(38,6)$ \\
& Viúvo & $26.160(30,5)$ \\
\hline & Separado/ divorciado & $8.005(9,3)$ \\
\hline & & \multicolumn{2}{c}{ Continua } \\
Raça/ cor & & $2.390(2,8)$ \\
& Ignorado & $45.119(52,6)$ \\
& Branca & $6.032(7,0)$ \\
& Preta & $486(0,6)$ \\
Total & Amarela & $33.523(39,1)$ \\
& Parda & $56(0,1)$ \\
& Indígena & $619(0,7)$ \\
& Ignorada & $85.835(100)$ \\
\hline
\end{tabular}

Fonte: Base de dados da Secretaria de Saúde do Distrito Federal.

As taxas de mortalidade apresentadas em forma de gráfico, na Figura 1, foram agrupadas pelos principais capítulos do CID 10, a saber capítulo I, J e C, que englobam respectivamente as causas cardiovasculares, pulmonares e neoplásicas. O gráfico das causas cardiovasculares indica um decréscimo na mortalidade do idosos, tanto homens quanto mulheres, com 80 anos ou mais, no período estudado. 
Research, Society and Development, v. 10, n. 17, e154101724503, 2021

(CC BY 4.0) | ISSN 2525-3409 | DOI: http://dx.doi.org/10.33448/rsd-v10i17.24503

Figura 1 - Gráfico de Coeficiente de mortalidade (por 10.000 habitantes) por faixa etária, causas específicas e sexo, por ano. Distrito Federal, Brasil, 2008 - 2018.

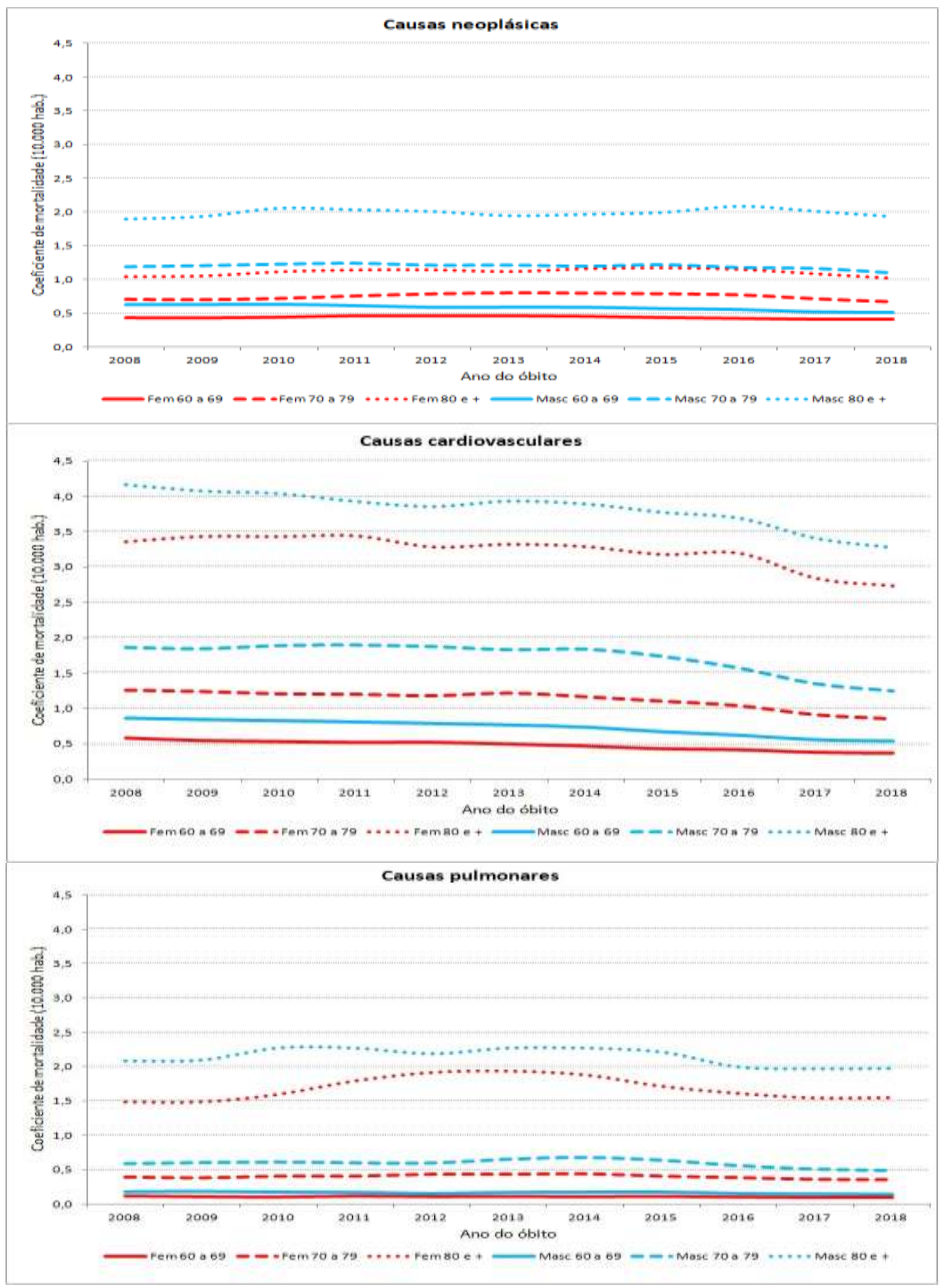

Fonte: Base de dados da Secretaria de Saúde do Distrito Federal.

Observa-se uma variação do coeficiente de mortalidade no período estudado. Houve queda na mortalidade de idosos, de ambos os sexos, de 60 a 79 anos, por causas cardiovasculares, pulmonares e neoplasias. Entretanto, verificou-se aumento da 
mortalidade nos idosos mais longevos. Na população de homens com 80 anos ou mais, ocorreu aumento de mortalidade por neoplasias. Entre as mulheres com 80 anos ou mais, houve aumento dos óbitos por causas pulmonares (Tabela 3).

Tabela 3 - Coeficiente de mortalidade (por 10.000 habitantes) e Taxa de variação (\%), por faixa etária e causas específicas, segundo sexo e ano. Distrito Federal, Brasil, 2008- 2018.

\begin{tabular}{llllll}
\hline & & \multicolumn{2}{l}{ Variação 2008/2018 $(\boldsymbol{\%})$} & \multicolumn{2}{l}{ Masculino } \\
Faixa etária & Causas de óbito & $\mathbf{2 0 0 8 / 2 0 1 8}$ & P-valor & $\mathbf{2 0 0 8 / 2 0 1 8}$ & P-valor \\
\hline De 60 a 69 anos & Cardiovasculares & $-37,31$ & $<0,0001^{*}$ & $-37,75$ & $<0,0001^{*}$ \\
& Pulmonares & $-15,48$ & 0,690 & $-21,52$ & 0,140 \\
& Neoplasias & $-4,13$ & $<0,0001^{*}$ & $-18,75$ & $0,007^{*}$ \\
De 70 a 79 anos & Cardiovasculares & $-32,77$ & $0,001^{*}$ & $-33,40$ & $<0,0001^{*}$ \\
& Pulmonares & $-9,56$ & 0,266 & $-16,33$ & 0,301 \\
80 anos e mais & Neoplasias & $-5,22$ & $0,007^{*}$ & $-7,79$ & $0,004^{*}$ \\
& Cardiovasculares & $-18,74$ & $<0,0001^{*}$ & $-21,44$ & $<0,0001^{*}$ \\
& Pulmonares & 4,24 & 0,056 & $-5,11$ & $0,013^{* *}$ \\
\hline
\end{tabular}

Fonte: Base de dados da Secretaria de Saúde do Distrito Federal * Estatisticamente significativo a 1\% ** Estatisticamente significativo a 5\%. Teste de comparação de proporções.

Em relação às taxas de mortalidade, houve um crescimento gradativo, conforme mostrado na Figura 2, no que diz respeito às faixas etárias e à proporção de óbitos. Quanto mais velhos os idosos, maior mortalidade (P-valor <0,001). Quanto ao sexo, o teste indicou que os homens apresentaram maior proporção de morte (P-valor <0,001). Com relação às causas do óbito, foi evidenciada a maior proporção de óbitos por causas cardiovasculares, porém não houve diferença entre causas pulmonares e neoplásicas (P-valor 0,006).

Figura 2 - Teste de tendência Jonckheere -Terpstra para coeficientes de mortalidade (por 10.000 habitantes) por faixa etária. Distrito Federal, Brasil, 2008-2018.

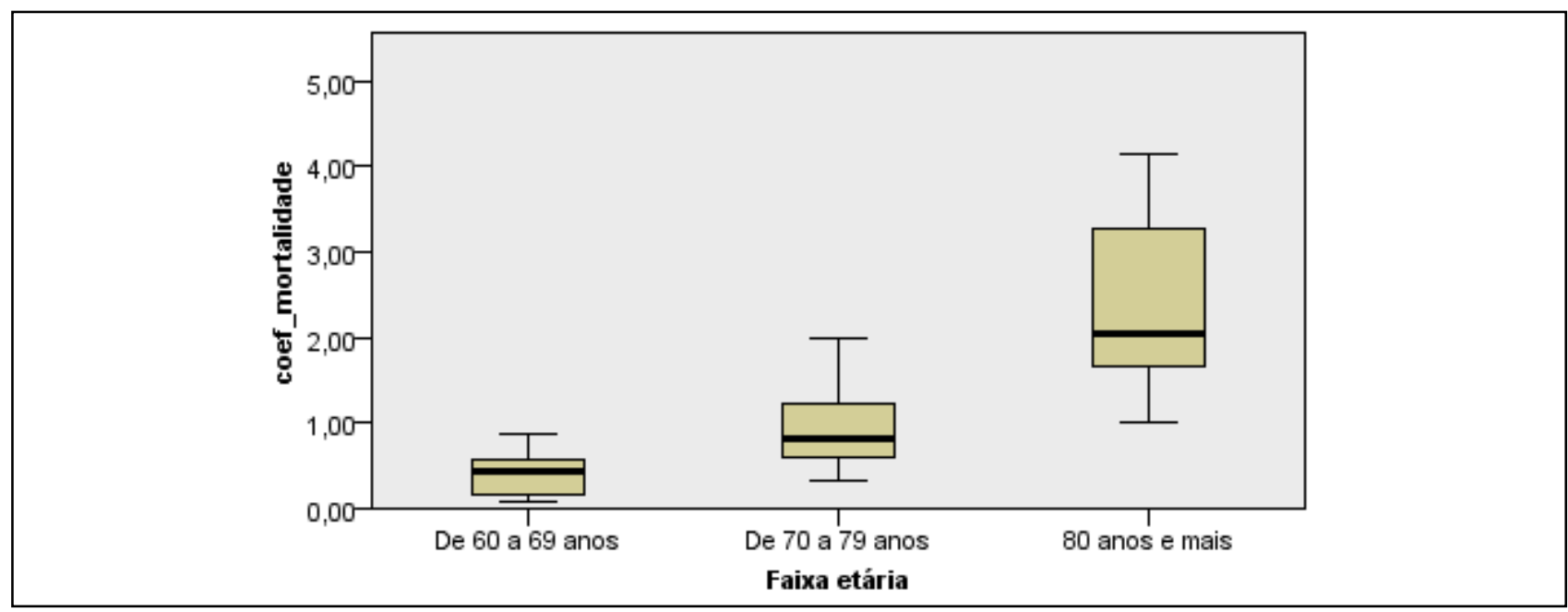

Fonte: Base de dados da Secretaria de Saúde do Distrito Federal.

\section{Discussão}

O presente estudo evidenciou predomínio de mortes entre idosos do sexo masculino. Os homens apresentaram maior proporção de morte, se comparados às mulheres. Dentre os idosos brasileiros, predominam as mulheres, nessa população foi 
demonstrado um risco de morte nos homens 1,73 vezes maior (Pereira et al., 2017). A maior mortalidade entre os homens pode ser explicada devido a características socioculturais, os quais, por vezes, postergam a busca do atendimento em saúde, tendendo a esperar o agravamento dos sintomas (Queiroz et al., 2018).

Ao analisar estratos etários dos idosos, o presente estudo identificou que quanto maior a idade maior o risco de morrer. Observa-se, diminuição da mortalidade geral dos idosos, o aumento da longevidade(Lima-Costa et al., 2004). O envelhecimento mundial tende a favorecer, assim como apresentado no presente estudo, a mortalidade em idades cada vez mais avançadas. Nesse contexto, a idade acima de 75 anos associou-se à maior mortalidade (Borim et al., 2017).

Quanto ao estado civil, a maioria dos óbitos ocorreu entre idosos casados. O presente estudo suscita questionamentos sobre a relação entre estado civil e mortalidade. Contudo essa associação não é simples, pois observa-se que indivíduos com melhores condições financeiras têm maior probabilidade de casar que aqueles que vivem em más condições econômicas e de saúde, tal fato pode justificar esse efeito protetor do matrimônio. Desse modo, o idoso casado tende a ter melhor situação econômica e a adotar melhores hábitos de vida (Manzoli et al., 2007).

Em relação à escolaridade dos idosos estudados, a maioria dos óbitos ocorreu em idosos com três anos ou menos de estudo. A baixa escolaridade está relacionada à maior mortalidade entre idosos, notadamente entre os homens que apresentam maior diferença na expectativa de vida, se comparados às mulheres (Silva et al., 2016).

O estudo mostrou também maior mortalidade entre idosos declarados brancos, contrastando com apenas $7 \%$ dos idosos falecidos declarados como negros. Nesse contexto, a miscigenação é uma característica histórica da formação da consolidação do povo brasileiro; a qual, contudo, pode interferir nas análises étnicas. Também o fato de a legislação brasileira considerar a raça autodeclarada permite interferência de fatores de difícil aferição. Considerações sobre a raça e as condições de saúde são complexas, uma vez que as disparidades observadas podem estar relacionadas tanto a questões biológicas quanto ao contexto social (Fontanarosa \& Bauchner, 2018).

Entre as principais causas de óbitos encontradas neste estudo, estão infarto do miocárdio e acidente vascular cerebral. No Brasil, essas doenças também constituem a principal causa de mortes dos idosos, e essa tendência tem se mantido em 1980, 1991, e 2000; entretanto, nesse período, foi observada uma diminuição das mortes por doenças cardiovasculares e um aumento daquelas causadas por neoplasias e por doenças do sistema respiratório (Lima-Costa et al., 2004).

Nesse contexto, as doenças do aparelho respiratório foram registradas como causa de grande percentual de óbitos, ocupando, no Distrito Federal, a segunda, a quarta e a quinta posições. No Brasil, em análises realizadas em 2005 e 2015, as doenças pulmonares, notadamente a doença pulmonar obstrutiva crônica (DPOC) e a pneumonia, estão entre as principais responsáveis pelos óbitos(Rossetto et al., 2019).

As neoplasias também apresentaram alta incidência dentre os óbitos. No Distrito Federal, o câncer de pulmão foi a quarta causa de mortalidade; citam-se ainda, entre as principais causas de morte, a neoplasia de próstata, de estômago, de mama e de cólon. No Nordeste e no Distrito Federal, o câncer é uma das principais patologias responsáveis pela morte de idosos. Entre as principais, estão câncer de próstata, de traqueia, de brônquios, de pulmões e de estômago(Carvalho \& Paes, 2019a). Dessa forma, nota-se, em diferentes contextos, a representatividade das neoplasias na mortalidade geral. Tal fato pode ser justificado, uma vez que, ao longo da vida, as células são progressivamente expostas a injúrias externas bem como o envelhecimento celular, o que favorece o surgimento de neoplasias (Nodhturft et al., n.d.).

Ainda, entre as 10 principais causas de morte, foi identificada a diabetes mellitus. A Pesquisa Nacional por Amostras de Domicílios (PNAD) de 2003 mostrou, entre os brasileiros de 70 a 79 anos, prevalência de 12\% em homens e de16\% em mulheres(Barros et al., 2006). Além das doenças crônico-degenerativas, as doenças infectocontagiosas também foram responsáveis por importante percentual de mortes. Nota-se a doença de chagas com comprometimento cardíaco sendo a nona 
maior causadora de mortes na presente série temporal. Essa doença é por vezes negligenciada, porém segue com elevada prevalência na população brasileira(Carlos Pinto Dias et al., 2016).

A presente investigação identificou queda de mortalidade para homens e mulheres idosos, por causas cardiovasculares, em todos os estratos etários. No Acre, também foi observada tendência à queda da mortalidade, por causas cardiovasculares(Bezerra et al., 2018); essa redução na mortalidade vem sendo notada no Brasil para todas as faixas etárias. Entre os anos de 1998-2012, observou-se no Sudeste queda de 61,9\% da mortalidade. Nesse mesmo período, na região Centrooeste, verificou-se queda da mortalidade de $26,9 \%$. Ressalta-se que a região Nordeste apresentou crescimento de mortalidade de 13,7\% (Guimarães et al., 2015).

A redução na mortalidade por causas cardiovasculares pode ser explicada pela melhora dos hábitos de vida. Além disso, o aumento da oferta de serviços de saúde, especialmente aqueles focados em prevenção e em promoção de saúde, aliado à melhor situação social e econômica do país, contribuem para essa tendência(Schmidt et al., 2011)(Schmidt et al., 2011). Apesar de os estudos evidenciarem diminuição na mortalidade por doenças cardiovasculares, a prevalência tem aumentado gradualmente. Assim, pode-se inferir melhor manejo e controle das doenças cardiovasculares, bem como dos fatores de risco (Ferreira et al., 2019).

As mortes ocasionadas por doenças pulmonares apresentaram queda. Estudo realizado em Santa Catarina evidenciou aumento da prevalência de doenças pulmonares em idosos(Rodrigues et al., 2019). Grande parte das doenças pulmonares está relacionada ao tabagismo. Sabe-se que esse hábito é observado entre os idosos brasileiros, entretanto foi evidenciado que idosos mais velhos apresentam menor prevalência de tabagismo, se comparados aos mais jovens(Gonçalves et al., 2019). O hábito de fumar está associado à significativa piora da saúde e da qualidade de vida, o qual ainda é um reconhecido como um fator de risco para adoecimento, mortes prematuras, DPOC, câncer e outras doenças(De São José et al., 2017).

A diminuição da mortalidade por causas pulmonares pode ser atribuída à vacinação contra influenza. Alguns tipos de influenza causam infecções graves e podem causar morte, especialmente das populações de risco, A vacinação dos idosos tem sido associada à menor mortalidade(Bacurau et al., 2019).

Houve ainda queda na mortalidade por causas neoplásicas. Estudo realizado no Nordeste apresentou aumento na mortalidade por câncer(Guimarães et al., 2015). As neoplasias são doenças multifatoriais, assim analisar os padrões de mortalidade das populações é uma tarefa complexa. Tendência de $0,9 \%$ de queda de mortalidade por neoplasias na população brasileira foi evidenciada no período de 2000 a 2011(Malta et al., 2014).

O decréscimo na mortalidade geral dos idosos foi evidenciada neste estudo. No Distrito Federal, a atenção primária à saúde figura entre as principais políticas de saúde. Nesse contexto, o aumento da cobertura, especialmente nas regiões mais vulneráveis, é fundamental na melhoria das condições de saúde da população (Göttems et al., 2019), além das ações voltadas para a melhoria da qualidade de atenção. Esses resultados acompanham o padrão de saúde dos brasileiros devido ao aperfeiçoamento dos serviços, em especial, a atenção primária(Malta et al., 2014). Ações oferecidas nesse nível de atenção à saúde, notadamente aquelas direcionadas para grupos prioritários(Borim et al., 2017), acesso ao serviço, vacinação contra influenza, redução do tabagismo e melhora das condições socioeconômicas impactam na redução da mortalidade(Bacurau et al., 2019).

Os resultados encontrados permitem levantar hipóteses para os resultados encontrados. Entretanto, as características específicas da população do Distrito Federal, de formação, habitação, relações políticas e distribuição de renda limitam a possibilidade de generalizações dos achados, que, portanto, deverão ser feitas com cautela. Ademais, pesquisas realizadas em bases de dados secundárias podem ser afetadas pela má qualidade dos dados. Ressalta-se, no entanto, que, no Distrito Federal, os óbitos suspeitos ou com carência de dados são investigados possivelmente minimizando essa limitação. 


\section{Conclusão}

Conclui-se que os óbitos dos idosos, no Distrito Federal, no período estudado, ocorreram principalmente por doenças cardiovasculares. Esse grupo de doenças aparenta lento, porém constante decréscimo em suas taxas, durante o período estudado. Os homens idosos apresentaram maior risco de morte, se comparados às mulheres. O comportamento das taxas de mortalidade por faixa etária, apesar de não surpreender, denota o alcance das políticas públicas de saúde para esse grupo populacional no Distrito Federal. Possivelmente o aumento na cobertura de atenção primária, o aprimoramento do acesso ao serviço de saúde e a oferta de serviços direcionados para grupos prioritários contribuíram para o padrão observado. Tais inciativas devem ser fortalecidas, a fim de garantir a manutenção e o aprimoramento dos ganhos observados. Os achados observados nesse estudo poderão ser corroborados por estudos futuros.

\section{Referências}

Antunes, J. L. F., \& Cardoso, M. R. A. (2015). Uso da análise de séries temporais em estudos epidemiológicos. Epidemiologia e Serviços de Saúde, 24(3), 565-576. https://doi.org/10.5123/S1679-49742015000300024

Bacurau, A. G. de M., Ferraz, R. de O., Donalisio, M. R., \& Francisco, P. M. S. B. (2019). Mortality due to cerebrovascular disease among the elderly and vaccination against influenza: São Paulo State, Brazil, 1980-2012. Cadernos de Saude Publica, 35(2). https://doi.org/10.1590/0102-311x00145117

Barros, M. B. de A., César, C. L. G., Carandina, L., \& Torre, G. D. (2006). Desigualdades sociais na prevalência de doenças crônicas no Brasil, PNAD-2003. Ciência \& Saúde Coletiva, 11(4), 911-926. https://doi.org/10.1590/S1413-81232006000400014

Bezerra, P. C. de L., Monteiro, G. T. R., Bezerra, P. C. de L., \& Monteiro, G. T. R. (2018). Trends in overall mortality and from diseases of the circulatory system in elderly individuals in Rio Branco, Acre, 1980-2012. Revista Brasileira de Geriatria e Gerontologia, 21(2), 143-154. https://doi.org/10.1590/198122562018021.170128

Borim, F. S. A., Francisco, P. M. S. B., \& Neri, A. L. (2017). Sociodemographic and health factors associated with mortality in community-dwelling elderly. Revista de Saude Publica, 51, 42. https://doi.org/10.1590/S1518-8787.2017051006708

Carlos Pinto Dias, J., Novaes Ramos, A., Dias Gontijo, E., Luquetti, A., Aparecida Shikanai-Yasuda, M., Rodrigues Coura, J., Morais Torres, R., Renan da Cunha Melo, J., Antonio de Almeida, E., de Oliveira Jr, W., Carlos Silveira, A., Marcondes de Rezende, J., Scalabrini Pinto, F., Walter Ferreira, A., Rassi, A., Augusto Fragata Filho, A., Silvestre de Sousa, A., Correia Filho, D., Maria Jansen, A., \& Alves, R. V. (2016). II Consenso Brasileiro em Doença de Chagas, 2015. Epidemiologia e Serviços de Saúde, 25(21), 1-10. https://doi.org/10.5123/S1679-49742016000500002

Carvalho, J. B., \& Paes, N. A. (2019a). Taxas de mortalidade por câncer corrigidas para os idosos dos estados do Nordeste brasileiro. Ciência \& Saúde Coletiva, 24(10), 3857-3866. https://doi.org/10.1590/1413-812320182410.03612018

Carvalho, J. B., \& Paes, N. A. (2019b). Corrected cancer mortality rates for the elderly in the states of the brazilian northeast. Ciencia e Saude Coletiva, 24(10), 3857-3866. https://doi.org/10.1590/1413-812320182410.03612018

De São José, B. P., De Amorim Corrêa, R., Malta, D. C., De Azeredo Passos, V. M., França, E. B., Teixeira, R. A., \& Camargos, P. A. M. (2017). Mortalidade e incapacidade por doenças relacionadas à exposição ao tabaco no Brasil, 1990 a 2015. Revista Brasileira de Epidemiologia, 20 , 75-89. https://doi.org/10.1590/1980-5497201700050007

Distrito Federal | Cidades e Estados | IBGE. (n.d.). https://www.ibge.gov.br/cidades-e-estados/df/.html?

Drumond, B., Ângelo, J., Xavier, D. R., Catão, R., Gurgel, H., \& Barcellos, C. (2020). Dinâmica espaço-temporal da dengue no Distrito Federal, Brasil: ocorrência e permanência de epidemias. Ciência \& Saúde Coletiva, 25(5), 1641-1652. https://doi.org/10.1590/1413-81232020255.32952019

Duarte, E. C., \& Barreto, S. M. (2012). Transição demográfica e epidemiológica: a Epidemiologia e Serviços de Saúde revisita e atualiza o tema. Epidemiologia e Serviços de Saúde, 21(4), 529-532. https://doi.org/10.5123/S1679-49742012000400001

Ferreira, S. R. G., Chiavegatto Filho, A. D. P., Lebrão, M. L., Duarte, Y. A. de O., \& Laurenti, R. (2019). Doenças cardiometabólicas. Revista Brasileira de Epidemiologia $=$ Brazilian Journal of Epidemiology, 21Suppl 02, e180008. https://doi.org/10.1590/1980-549720180008.supl.2

Figueiredo, A. E. B., Ceccon, R. F., \& Figueiredo, J. H. C. (2021). Doenças crônicas não transmissíveis e suas implicações na vida de idosos dependentes. Ciência \& Saúde Coletiva, 26(1), 77-88. https://doi.org/10.1590/1413-81232020261.33882020

Fontanarosa, P. B., \& Bauchner, H. (2018). Race, Ancestry, and Medical Research. JAMA, 320(15), 1539-1540. https://doi.org/10.1001/JAMA.2018.14438

Gonçalves, I. B., Lebrão, M. L., Duarte, Y. A. de O., Wagner, G. A., \& Zanetta, D. M. T. (2019). Estado nutricional de idosos fumantes e ex-fumantes da cidade de São Paulo, Brasil. Revista Brasileira de Epidemiologia = Brazilian Journal of Epidemiology, 21Suppl 02, e180013. https://doi.org/10.1590/1980549720180013.supl.2

Göttems, L. B. D., Almeida, M. O. de, Raggio, A. M. B., Bittencourt, R. J., Göttems, L. B. D., Almeida, M. O. de, Raggio, A. M. B., \& Bittencourt, R. J. (2019). O Sistema Único de Saúde no Distrito Federal, Brasil (1960 a 2018): revisitando a história para planejar o futuro. Ciência \& Saúde Coletiva, 24(6), 1997-2008. https://doi.org/10.1590/1413-81232018246.08522019 
Guimarães, R. M., Andrade, S. S. C. de A., Machado, E. L., Bahia, C. A., Oliveira, M. M. de, \& Jacques, F. V. L. (2015). Diferenças regionais na transição da mortalidade por doenças cardiovasculares no Brasil, 1980 a 2012. Revista Panamericana de Salud Pública, 37, 83-89. http://www2.data

Lima-Costa, M. F., Peixoto, S. V., \& Giatti, L. (2004). Tendências da mortalidade entre idosos brasileiros (1980 - 2000). Epidemiologia e Serviços de Saúde, 13(4), 217-228. https://doi.org/10.5123/S1679-49742004000400004

Malta, D. C., Moura, L. de, Prado, R. R. do, Escalante, J. C., Schmidt, M. I., \& Duncan, B. B. (2014). Mortalidade por doenças crônicas não transmissíveis no Brasil e suas regiões, 2000 a 2011. Epidemiologia e Serviços de Saúde, 23(4), 599-608. https://doi.org/10.5123/S1679-49742014000400002

Manzoli, L., Villari, P., M Pirone, G., \& Boccia, A. (2007). Marital status and mortality in the elderly: A systematic review and meta-analysis. Social Science \& Medicine, 64(1), 77-94. https://doi.org/10.1016/J.SOCSCIMED.2006.08.031

Nodhturft, V., Schneider, J. M., Hebert, P., Bradham, D. D., Bryant, M., Phillips, M., Russo, K., Goettelman, D., Aldahondo, A., Clark, V., \& Wagener, S. (n.d.). CHRONIC DISEASE SELF-MANAGEMENT Improving Health Outcomes. Retrieved May 6, 2019, from https://www.researchgate.net/profile/Douglas_D_Bradham/publication/12446184_Chronic_disease_self-

management_Improving_health_outcomes/links/0deec51df143057968000000/Chronic-disease-self-management-Improving-health-outcomes.pdf

Paes, N. A. (2016). A Geografia Da Mortalidade Por Causas Dos Idosos No Brasil. Anais, O(0), 1-22. http://www.abep.org.br/publicacoes/index.php/anais/article/view/1007

Pereira, A. A., Borim, F. S. A., \& Neri, A. L. (2017). Ausência de associação entre o índice de fragilidade e a sobrevivência de idosos no Brasil: Estudo FIBRA. Cadernos de Saúde Pública, 33(5). https://doi.org/10.1590/0102-311x00194115

Queiroz, T. S., Rehem, T. C. M. S. B., Stival, M. M., Funghetto, S. S., Lima, L. R. de, Cardoso, B. G., Santos, W. S., Queiroz, T. S., Rehem, T. C. M. S. B., Stival, M. M., Funghetto, S. S., Lima, L. R. de, Cardoso, B. G., \& Santos, W. S. (2018). How do old men take care of their own health in Primary Care? Revista Brasileira de Enfermagem, 71(suppl 1), 554-561. https://doi.org/10.1590/0034-7167-2017-0131

Rodrigues, M. M., Alvarez, A. M., \& Rauch, K. C. (2019). Tendência das internações e da mortalidade de idosos por condições sensíveis à atenção primária. Revista Brasileira de Epidemiologia, 22, e190010. https://doi.org/10.1590/1980-549720190010

Rossetto, C., Soares, J. V., Brandão, M. L., Rosa, N. G. da, \& Rosset, I. (2019). Causes of hospital admissions and deaths among Brazilian elders between 2005 and 2015. Revista Gaucha de Enfermagem, 40, e20190201. https://doi.org/10.1590/1983-1447.2019.20190201

Schmidt, M. I., Duncan, B. B., e Silva, G. A., Menezes, A. M., Monteiro, C. A., Barreto, S. M., Chor, D., \& Menezes, P. R. (2011). Chronic noncommunicable diseases in Brazil: burden and current challenges. The Lancet, 377(9781), 1949-1961. https://doi.org/10.1016/S0140-6736(11)60135-9

Silva, L. E. da, Freire, F. H. M. de A., Pereira, R. H. M., Silva, L. E. da, Freire, F. H. M. de A., \& Pereira, R. H. M. (2016). Diferenciais de mortalidade por escolaridade da população adulta brasileira, em 2010. Cadernos de Saúde Pública, 32(4). https://doi.org/10.1590/0102-311X00019815

Veras, R. (2016). Linha de cuidado para o idoso: detalhando o modelo. Revista Brasileira de Geriatria e Gerontologia, 19, 887-905. https://doi.org/10.1590/1981-22562016019.160205 